\title{
Side-Pinch Effect of a Magnetically Driven Shock Tube with Parallel Plate Electrodes
}

\section{Chang, C. T.; Korsbech, Uffe C C; Mondrup, K.}

Published in:

Physics of Fluids

Link to article, DOI:

$10.1063 / 1.1692633$

Publication date:

1969

Document Version

Publisher's PDF, also known as Version of record

Link back to DTU Orbit

Citation (APA):

Chang, C. T., Korsbech, U. C. C., \& Mondrup, K. (1969). Side-Pinch Effect of a Magnetically Driven Shock Tube with Parallel Plate Electrodes. Physics of Fluids, 12(Supplement I), I-73-I-75. https://doi.org/10.1063/1.1692633

\section{General rights}

Copyright and moral rights for the publications made accessible in the public portal are retained by the authors and/or other copyright owners and it is a condition of accessing publications that users recognise and abide by the legal requirements associated with these rights.

- Users may download and print one copy of any publication from the public portal for the purpose of private study or research.

- You may not further distribute the material or use it for any profit-making activity or commercial gain

- You may freely distribute the URL identifying the publication in the public portal 


\title{
Side-Pinch Effect of a Magnetically Driven Shock Tube with Parallel Plate Electrodes
}

\author{
C. T. Chang, U. Korsbech, and K. Mondrup \\ Atomic Energy Commission, Research Establishment Risö, Roskilde, Denmark, and the \\ Technical University of Denmark, Copenhagen, Denmark
}

\begin{abstract}
To study the possible effect of the side pinch on the steady-state current and the steady-state shock speed of a magnetically driven shock tube, a semiempirical model is formulated. The time history of the current, the radial and the translational motion of the current-carrying region are expressed by three interacting nonlinear equations with five adjustable parameters describing the variation of the electric circuit elements, the geometry of the shock tube, and the initial running conditions. Within the range of practical interest for values of the parameters investigated, computational results show that the current-carrying region oscillates radially, but the current and the translational velocity of the current-carrying region approach the quasi-steady state rapidly. The deviation of the current from its "steady-state" value (when the pinch effect is absent) in no case amounts to more than $5 \%$.
\end{abstract}

\section{INTRODUCTION}

From the point of view of the electric circuit, the motion of the current sheet along the electrodes of a magnetically driven shock tube causes the inductance of the electrodes to vary with time. This implies that it has a feedback effect on the driving current.

In a previous paper, ${ }^{1}$ taking a broad-rail gun as a special example, we have shown that when a battery is chosen as an energy source, regardless of the value of its internal resistance, the current quickly approaches a steady-state value; the current sheet, after a relatively short accelerating period, moves at a constant speed. In the analytical model used, one of our main assumptions is that the field is uniform behind the current sheet. This assumption is reasonable so long as the width of the electrodes is much greater than the distance travelled by the current sheet, but certainly necessitates a careful re-examination when they are of comparable magnitude.

The purpose of the present work is to investigate to what extent the finite width of the electrodes influences the steady state of the driving current and the motion of the current sheet.

It should be mentioned that our treatment is in many respects similar to that contained in a paper by Kolesnikov. ${ }^{2}$ However, it differs from his on the following points: (a) His energy source is a fastdischarge condenser bank, ours is a constant-voltage battery; (b) the current-carrying plasmoid in his case moves in a vacuum, in our case in a medium

${ }^{1}$ C. T. Chang and O. Kofoed-Hansen, Plasma Phys. 10, 137 (1968).

2 P. M. Kolesnikov, Zh. Tekh. Fiz. 35, 1577 (1965) [Sov. Phys.-Tech. Phys. 10, 1219 (1966)].

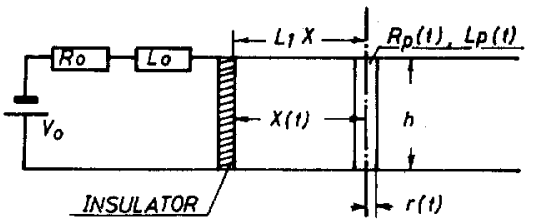

FIG. 1. Schematic drawing of the electric circuit and the shock tube.

compressed by the shock; (c) the resistance of the plasmoid is neglected in his treatment, but not in ours.

\section{ANALYTICAL MODEL}

As in our previous treatment, we assume the plasmoid to be accelerated between two parallel plane electrodes of width $b$ and a distance $h$ apart. The energy source is a battery of voltage $V_{0}$ and internal resistance $R_{0}$. The electrodes, the battery, and the connecting electric circuit are shown schematically in Fig. 1.

To carry out the analysis, we make the following assumptions:

(i) Once the electric breakdown occurs, the current is confined within a cylindrical column of constant mass (i.e., a plasmoid).

(ii) The associated magnetic field is distributed in such a way that the field lines are more crowded in the rear and at the sides of the cylinder than in the front. This causes the plasmoid to move forward as a pulsating hypersonic projectile and produces a shock in front of it.

(iii) In reality, the cross section of the cylinder probably closely resembles an elongated ellipse. For simplicity, in considering the pinch effect, we take the plasmoid to be a circular cylinder.

(iv) The temperature and pressure of the plasma 
within the cylinder vary with its radius in accordance with the adiabatic compression law.

(v) The electrical resistance of the plasmoid is given by the usual formula for a fully ionized gas $^{3}$; thus, it depends mainly on the temperature and can be written as

$$
R_{p}=R_{p}^{*}\left(\frac{r}{b / 2}\right)^{3 \gamma-5}
$$

where $R_{p}^{*}$ is the resistance of the plasmoid during the breakdown.

(vi) The inductance of the circuit is made up of the lead inductance $L_{0}$, the variable inductance of the electrodes $L_{e}=L_{1} x_{(t)}$, where $L_{1}$ is the inductance per unit length of the electrodes and $x_{(t)}$ is the distance traveled by the center of mass of the plasmoid, and the variable inductance of the pulsating plasmoid $L_{p}=(\mu h / 2 \pi) \ln \left[\left(\frac{1}{2} b\right) / r\right]$.

(vii) In estimating the mass swept up by the shock, we adopt the "snowplow" model. To account for the fact that the plasmoid is pulsating, we introduce a numerical factor $\alpha(\leq 1)$. The mass $M$ swept up by the shock and accumulated ahead of the projectile is then $M=\alpha \rho_{0} b h x$, where $\rho_{0}$ is the initial gas density. When the pinch effect is not very pronounced, $\alpha$ can be taken to be time-independent.

On these assumptions, the governing equations in their dimensionless form can be written as follows: voltage balance,

$$
\begin{aligned}
& 1-\xi-\frac{d \xi}{d \tau}-\frac{d}{d \tau}(\eta \xi) \\
& \quad=A_{2} \xi \nu^{3 \gamma-5}-A_{1} \frac{d}{d \tau}(\xi \ln \nu) ;
\end{aligned}
$$

momentum equation of the translational motion of the plasmoid,

$$
\epsilon^{*^{2}} \xi^{2}=\frac{d^{2}}{d \tau^{2}}\left(\frac{\eta^{2}}{2}\right)
$$

momentum equation of the radial oscillation of the plasmoid,

$$
\frac{d^{2} \nu}{d \tau^{2}}=-A_{3} \frac{\xi^{2}}{\nu}+A_{4}\left(\frac{1}{\nu}\right)^{2 \gamma-1}
$$

In the above, the dimensionless variables are defined as

$$
\tau=\frac{\tilde{u}}{x_{0}} \frac{t}{\epsilon}, \quad \xi=\frac{I}{I_{0}}, \quad \eta=\frac{x}{x_{0}}, \quad \nu=\frac{r}{(b / 2)} ;
$$

the scaling factors are

$$
\text { length of translational motion, } x_{0}=L_{0} / L_{1} \text {, }
$$

$$
\text { length of radial oscillation, } \frac{1}{2} b \text {, }
$$

${ }^{3}$ L. Spitzer, Jr., Physics of Fully Ionized Gases (Interscience Publishers, Inc., New York, 1962), 2nd ed. velocity,

$$
\tilde{u}=\left(\frac{\mu}{2 \rho_{0} b^{2}}\right)^{1 / 2} \frac{V_{0}}{R_{0}},
$$

current,

$$
I_{0}=V_{0} / R_{0} .
$$

The parameter $\epsilon^{*}$ differs from $\epsilon$ used previously ${ }^{1}$ only by the "sweeping" factor $\alpha$; thus $\epsilon^{*}=\epsilon / \alpha^{1 / 2}$; the remaining parameters, $A_{1}, A_{2}, A_{3}$, and $A_{4}$ are defined as

$$
\begin{array}{ll}
A_{1}=\frac{\mu h}{2 \pi L_{0}}, & A_{2}=\frac{R_{p}^{*}}{R_{0}}, \\
A_{3}=\epsilon^{2}\left(\frac{t_{a}}{t_{c}}\right)^{2}, & A_{4}=\beta A_{3},
\end{array}
$$

where $t_{a}=x / \tilde{u}$ is a time scale related to the accelerating period of the translational motion of the plasmoid, $t_{c}=\left(2 \pi / I_{0}\right)\left(\frac{1}{2} b\right)^{2}\left(\rho_{0} / \mu\right)^{1 / 2}$ is a time scale related to the "collapsing time" of the pinch, and $\boldsymbol{\beta}=p_{0} /\left(p_{m}\right)_{0}\left[p_{0}\right.$ is the initial gas pressure and

$$
\left.\left(p_{m}\right)_{0}=\frac{\mu I_{0}^{2}}{2 \pi^{2} b^{2}}\right] \text {. }
$$

One must recall that the pinch effect does not come into play until $\tau>\tau_{1}$. This implies that Eq. (4) is valid only for $\tau>\tau_{1}$. For $\tau \leq \tau_{1}$ it must be replaced by

$$
\nu \equiv 1 \text {. }
$$

The time instant $\tau_{1}$ clearly is to be determined from the condition

$$
A_{3} \xi^{2}\left(\tau_{1}\right)=A_{4} \quad \text { or } \quad \xi^{2}\left(\tau_{1}\right)=\beta,
$$

which means that the current has reached the value at which the magnetic pressure just balances the gas pressure.

To start the computation we insert $\nu=1$ in Eq. (1) in combination with the initial condition

$$
\eta(0)=0, \quad \xi(0)=0, \quad \eta(0)\left(\frac{d \eta}{d \tau}\right)_{\tau=0}=0,
$$

and use Eqs. (2) and (3) to compute $\xi$ and $\eta$ up to the moment $\tau=\tau_{1}$. From that moment onward, the computation is continued with the full set of equations (4), (2), and (3) in combination with the condition

$$
\nu\left(\tau_{1}\right)=1, \quad\left(\frac{d \nu}{d \tau}\right)_{\tau=\tau_{1}}=0
$$

\section{A. Computational Result}

Using the starting procedure outlined in the preceding section, we analyze the system of equations (2), (3), and (4) numerically. In accordance with the geometry of the gun and the capacity of the battery used in our experiments, the values of the param- 


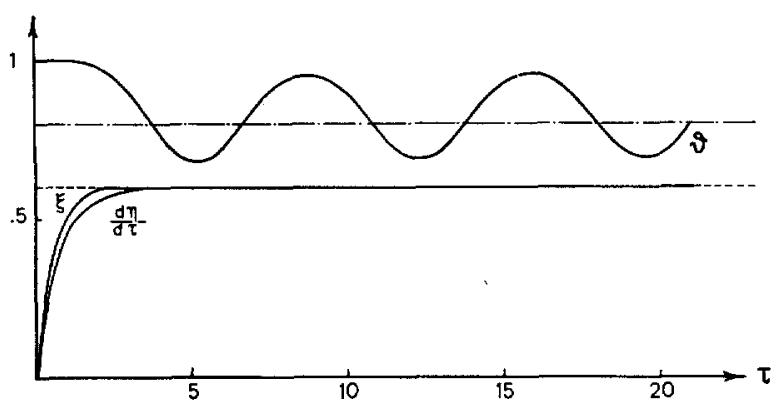

FIG. 2. Solutions of $\nu, \xi$, and $d \eta / d \tau$ corresponding to the "sinusoidal" mode. Dashed line, "asymptotic" value of $\xi=\hat{\xi}$. Broken line, "asymptotic" value of $\nu=\hat{\nu}$. Values of parameters used: $\gamma=\frac{5}{3}, \epsilon^{*}=1.0, A_{1}=0.2, A_{2}=0.04$, $A_{3}=1.0, A_{4}=0.27$.

eters are chosen to cover the following ranges:

$$
\begin{gathered}
0.1 \leq \epsilon^{*} \leq 5, \quad A_{1}=0.2, \quad A_{2}=0.04, \\
0.01 \leq A_{3} \leq 25, \quad 0.0003 \leq A_{4} \leq 1.96 .
\end{gathered}
$$

Specifically, they are chosen in this way: for each assigned value of $\epsilon^{*}$ we calculate $A_{3}$ (for the gun and the battery used in our experiment, $\left.A_{3} \sim \epsilon^{*^{2}}\right)$; then, we select five different values of $A_{4}$ so that the "asymptotic" value of $\hat{\nu}$ falls in the range of $0.1 \leq$ $\hat{\nu} \leq 0.9$.

Generally speaking, the computational result shows that the radial motion of the plasmoid can be classified into two types: that resembling a "sinusoidal oscillation" and that resembling a "linear pinch." The former mode usually appears for a combination of large values of $\epsilon^{*}$ and $\beta$, the latter mode for small values of $\epsilon^{*}$ and $\beta$. Typical examples of solutions corresponding to these two different modes are shown in Figs. 2 and 3, respectively. It is interesting to note that in both cases the current and the speed of the plasmoid approach nearly constant values after a relatively short accelerating period.

An explanation is required of the meaning of the "asymptotic" values $\hat{\nu}, \hat{\xi}$, and $d \hat{\eta} / d \tau$. Clearly, as the oscillation of the plasmoid implies that the

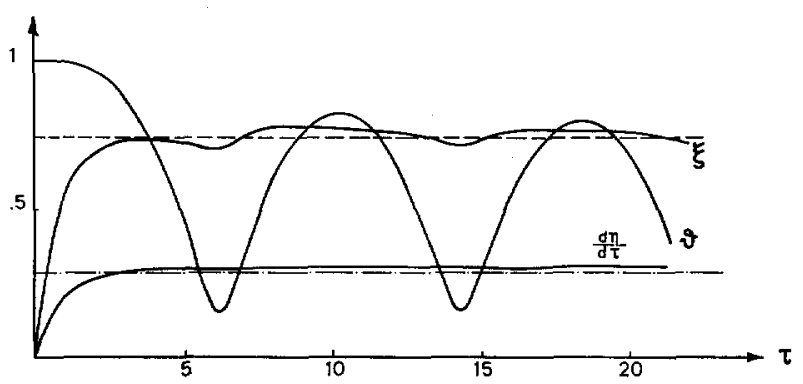

FIa. 3. Solutions of $\nu, \xi$, and $d \eta / d \tau$ corresponding to the "pinch" mode. Dashed line, "asymptotic" value of $\xi=$ $\xi$. Broken line, "asymptotic" value of $\nu=\hat{\nu}$. Values of parameters used: $\gamma=\frac{5}{3}, \epsilon^{*}=0.4, A_{1}=0.2, A_{2}=0.04$, $A_{3}=0.16, A_{4}=0.016$.

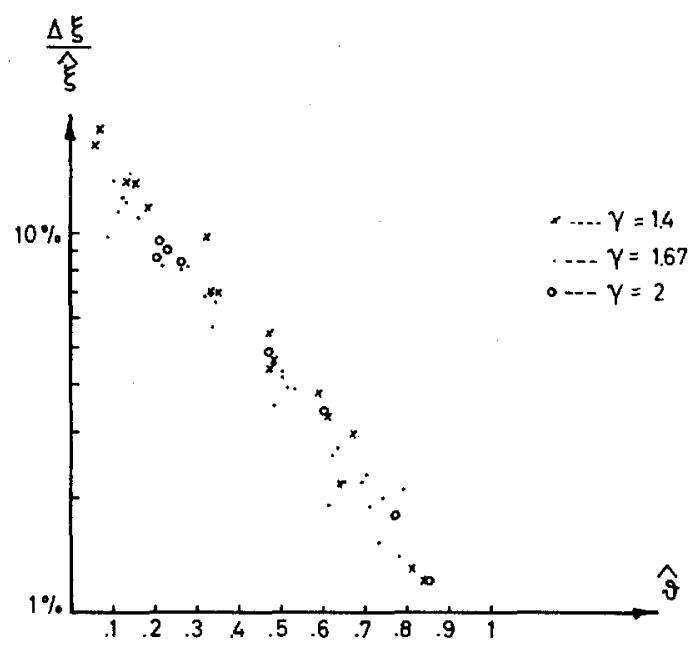

FIG. 4. Variation of the current $\Delta \xi / \hat{\xi}$ with respect to the "asymptotic" value $\hat{\nu}$.

plasmoid inductance $L_{p}$ varies with time, strictly speaking there are no steady states for these variables. However, to establish some standard of reference we can easily solve for those values of $\nu, \xi$, and $d \eta / d \tau$ by putting $d \nu / d \tau=0, d \xi / d \tau=0$, and $d^{2} \eta / d r^{2}=0$ in Eqs. (2), (3), and (4), and call the solutions "asymptotic" values for convenience.

\section{B. Discussion and Remarks}

The numerical computation also gives the very interesting result that the current variation $\Delta \xi / \hat{\xi}$ depends essentially on the "asymptotic" value of $\hat{\nu}$, where $\Delta \xi$ is defined as the value of $\xi$ at the second maximum minus the value of $\xi$ at the succeeding minimum. In other words, all combinations of $A_{3}$, $A_{4}$, and $\gamma$ which give the same value of $\hat{\nu}$ also give the same percentage variation of $\Delta \xi / \hat{\xi}$ (see Fig. 4). Physically, this might be due to the fact that the resistance $R_{p}$ of the plasmoid is negligible; the current variation depends essentially on the parameter $\epsilon^{*}$.

We might also remark here that in our computational work we have on purpose avoided very low values of $A_{4}$ (or $\beta$ ) for the following reasons:

(a) A smaller value of $\beta$ causes a stronger pinch, which might introduce some hydromagnetic instabilities.

(b) A too small value of $\beta$ causes a too small value of $\hat{\nu}$, which leads to a numerical instability in the computational work.

(c) A smaller value of $\beta$ causes a stronger oscillation of the plasmoid, which might violate the assumption that the "sweeping" factor $\alpha$ is timeindependent. 